\title{
SENSIBILIZACIÓN Y EXPOSICIÓN A PÓLENES Y ESPORAS DE HONGOS ALERGÉNICOS AEROTRANSPORTADOS EN CIUDADES DEL SUR DE PERÚ
}

\author{
SENSITIZATION AND EXPOSURE TO AIRBORNE POLLENS AND \\ FUNGAL SPORES IN SOUTH PERUVIAN CITIES
}

${ }^{1}$ Oscar Manuel Calderón Llosa, ${ }^{2}$ Silvia Antonieta Uriarte Obando, ${ }^{3}$ Gregorio Pedro Tejada Monroy

\section{RESUMEN}

Introducción y objetivos: Es muy importante el conocimiento de las fluctuaciones estacionales y anuales sobre los pólenes y esporas de hongos alergénicos aerotransportados en cualquier área geográfica. Nuestro objetivo fue identificar los aeroalérgenos más importantes en la atmósfera de la ciudad urbana de Tacna a los cuales la población está expuesta y desarrollar un estudio alergológico para establecer perfiles de sensibilización en las ciudades de Tacnay Arequipa. Material y métodos: El conteo polínico y fúngico se realizó de acuerdo a la técnica estandarizada con un equipo Burkard spore trap for 7 days (Burkard manufacturing ${ }^{\circledR}$, Herst, United Kingdom) y el procedimiento de análisis recomendado por el comité de aerobiología de la Sociedad Española de Alergología e Inmunología Clinica. El estudio alergológico consistió en anamnesis y pruebas cutáneas con extractos (ALK-abello *, Madrid-España). Resultados: Los 3 tipos de esporas de hongos más relevantes que se encontraron durante el periodo de muestreo en orden de abundancia fueron: Cladosporium herbarum (47,22\%), Alternaria alternata (33\%), Nigrospora spp (19,8\%). También encontramos 4 taxones polinicos: Oleacea (94,7\%), Chenopodiaceae-Amaranthaceae (3\%), Poaceae $(2,08 \%)$, Myrtaceoe (Eucalipto) $(0,77 \%)$. Perfil de sensibilización al polen de Olea europea, en muestras aleatorias en las ciudades de Tacna (40\%) y Arequipa (36\%) y a Alternaria alternata (4\%) y (8\%) respectivamente. Conclusiones: Éste es el primer estudio realizado en Perú sobre sensibilización y concentraciones de granos de polen y esporas de hongos medidos por método volumétrico. Sugerimos ampliar estudios e implementar estaciones de aerobiología que provean mayor información y sirvan como guía para una mejor prevención, diagnóstico y tratamiento para la población de la zona sur de Perú conenfermedades alérgicas

Palabras claves: Polinosis, Polen de Oleaeuropaea, esporas de hongos, Alternario alternata, rinitis y/o asma, aerobiologia, Tacna, Arequipa.

\section{ABSTRACT}

Introduction and objectives: Is very important the knowledge about seasonal and annual fluctuations in airbome pollen and fungal spores in any geographical area. Our objective was to identify the most impor tant aeroallergens in the atmosphere of Tacna urban city to which the population is exposed, and perform an allergological study for establish sensitization profile in Tacna and Arequipa cities. Material and methods: The pollen and fungal spores counts were made according to standardized technique with Burkard spore trap for 7 days (Burkard Manufacturing ${ }^{\circledR}$, Herst, United Kingdom) and the analysis procedures recommended by the aerobiology committee of the Spanish Society of Allergology and Clinical Immunology. The allergological study consisted in anamnesis and skin prick tests (ALK-abello $B_{1}$, Madrid-España). Results: The 3 most relevant fungal spores during all the period of sampling, in order of abundance, were: Cladosporium herbarum (47,22\%), Alternaria alternata (33\%), Nigrospora spp (19,8\%). We also found 4 pollens taxa: Oleaceae $(94,1 \%)$. Chenopodiaceae-Amaranthaceae (3\%), Poaceae $(2,08 \%)$, Myrtaceoe (Eucalyptus) $(0,77 \%)$. Profile of sensitization to Olea europea pollen in a random population in Tacna (40\%) and Arequipa (36\%) cities and to Aiternaria olternata $(4 \%)$ and $(8 \%)$ respectively. Conclusions: This is the first study in Perú about sensitization and concentration of airborne pollen and fungal spores measured with a volumetric method. We suggest further research studies and to implement aerobiological stations that provide information to guide us to a good prevention, diagnosis and treatment to the peruvian south population with allergy diseases.

Keywords: Pollinosis, Olea europaea pollen, fungal spores, Alternaria alternata, rhinitis, asthma, aerobiology, Tacna, Arequipa.

\section{INTRODUCCIÓN}

Es muy importante el conocimiento de las fluctuaciones estacionales y anuales sobre los pólenes y esporas de hongos alergénicos aerotransportados en cualquier área geográfica. Es esencial para el diagnóstico y tratamiento efectivo de las enfermedades alérgicas.
Nuestro objetivo fue identificar los aeroalérgenos más importantes en la atmósfera de la ciudad urbana de Tacna, a los cuales la población está expuesta, y desarrollar un estudio alergológico para establecer perfiles de sensibilización en la población de las ciudades de Tacna y Arequipa de la zona sur de Perú.

\footnotetext{
'Especialista en Alergología e Inmunología Clínica. Hospital Universitario La Paz - Instituto de investigación (IdiPAZ), Departamento de Alergolo-' gia.Madrid, España.

${ }^{2}$ Médico alergóloga. Fundación Jiménez Díaz, Departamento de Alergologia. Madrid, España.

${ }^{3}$ Doctor en Ciencias Ambientales, Ingeniero Quimico. Docente Principal de la Universidad Nacional Jorge Basadre Grohmann. Tacna, Perú.
} 
Calderón, $\mathbf{O}$. et al. Sensibilización y exposición a pólenes y esporas de hongos alergénicos aerotransportados en ciudades del sur de Perú.

\section{MATERIAL Y MÉTODOS}

El conteo polínico y fúngico se realizó de acuerdo a la técnica estandarizada con un equipo Burkard spore trap for 7 days (Burkard manufacturing ${ }^{\circledR}$, Herst, United Kingdom) y el procedimiento de análisis recomendado por el comité de acrobiología de la Socicdad Española de Alergología e Inmunologia Clínica. El equipo se instaló en el área urbana de Tacna, en la azotea de un edificio de $20 \mathrm{~m}$ de altura, el periodo de muestreo se desarrolló entre octubre y diciembre del 2014 (estación de primavera). Fl estudio alergológico consistió en anamnesis y pruebas cutáneas. En la ciudad de Tacna se realizó en un grupo aleatorio de 100 trabajadores voluntarios de las entidades públicas (Migraciones y Promperu), rango de edad: 20-80 años, y en la ciudad de Arequipa el estudio alergológico se realizó en un grupo aleatorio de 100 personas voluntarias, rango de edad 4-70 años. En ambas muestras, la población en estudio era natural de cada ciudad y vivían actualmente en ellas.

Las pruebas cutáneas se realizaron con alérgenos estandarizados de Olea europaea, Lolium perenne, Alternaria alternata, Cladosporium berbarum, Penicilium notatum, Aspergillus fumigatus (ALK-abelloß, Madrid-España), scgún las guías recomendadas por Bousquet J. et al. ${ }^{6}$

\section{RESULTADOS}

Los 3 tipos de esporas de hongos más relevantes que se encontraron durante el periodo de muestreo en orden de abundancia fueron: Cladosporium berbarum $(47,22 \%)$, Alternaria alternata (33\%), Nigrospora spp (19,8\%). Encontramos un pico importante de Alternaria alternata $\left(77\right.$ esporas $\left./ \mathrm{m}^{3}\right)$ el 31 de octubre y en la siguiente semana los conteos estuvieron entre (60-70 esporas $\left./ \mathrm{m}^{3}\right)$. Ver figura 1 .

Tabla 1. Conteo total, conteo promedio en la semana pico, semana pico.

\begin{tabular}{|c|c|c|c|}
\hline & $\begin{array}{l}\text { Conteo total } \\
\left.\text { (Esporas } / \mathrm{m}^{3}\right)\end{array}$ & $\begin{array}{l}\text { Conteo promedio } \\
\text { en la semana pico }\end{array}$ & Semana pico \\
\hline Alternaria alternala & 372 & 40 & Octubre $-4^{n}$ \\
\hline Nigrospara spp. & 646 & 15 & Octubrc $-3^{n}$ \\
\hline Cladosporium b. & 911 & 35 & Diciembre $-1^{\#}$ \\
\hline
\end{tabular}

También encontramos 4 taxones polínicos: Oleaced (94,1\%), Chenopodiaceae-Amaranthaceae (3\%), Poaceae $(2,08 \%)$, Myrtaceae (Eucalipto) $(0,77 \%$ ). Los valores más elevados de Olea europea se registraron entre la segunda y tercera semanas de octubre, con conteos diarios entre 60 hasta 125 gra$\mathrm{nos} / \mathrm{m}^{3}$ por día, y descenso entre noviembre y diciembre, 40 granos $/ \mathrm{m}^{3}$ o menos. Figura 2 .

\section{Estudio alergológico}

En la ciudad de Tacna encontramos 14 sujetos sensibilizados al extracto de I olium perenne (14\% del total testado de la población) y 40 personas al extracto de Olea europaea $(40 \%), 14$ estuvieron sensibilizados a ambos pólenes. Sobre el grupo sensibilizado a Olea europaea, $85 \%$ de ellos referían alguna sintomatologia como rinitis, rinoconjuntivitis $y / o$ asma en algún momento del año, sin clara estacionalidad o que podía ser desencadenado por la exposición al polvo doméstico.

Sin embargo, $25 \%$ de las personas sensibilizadas al polen de Olea europaea tuvieron rinoconjuntivitis y 5\% asma en primavera. También encontramos sensibilización a Alter. naria alternata en 4 pacientes, uno de ellos sensibilizado también a Olea europea.

En la ciudad de Arequipa encontramos sensibilización al extracto de Lolium perenne en 12 personas $(12 \%$ del total testado de la población) y 36 personas al extracto de Olea europaea $(36 \%), 12$ estuvieron sensibilizadas a ambos pólenes. Se encontró sensibilización a Alternaria alternata en 8 pacientes $(8 \%) .50 \%$ del total del grupo en estudio presentó rinitis, $5 \%$ asma y $3 \%$ rinitis y asma, muchos de ellos presentaron sintomas perennes, no referían claros desencadenantes. No se detectó sensibilización a otros alérgenos testados en las ciudades estudiadas.

Tabla 2. Conteo total, conteo promedio en la semana pico, semana pico.

\begin{tabular}{lccc}
\hline & $\begin{array}{c}\text { Conteo total } \\
\left(\text { Granos } / \mathrm{m}^{3}\right)\end{array}$ & $\begin{array}{c}\text { Conteo promedio } \\
\text { en la semana pico }\end{array}$ & Semana pico \\
\hline Olea esuropaea (olivo) & 1220 & 90 & Octubre $-2^{4}-3^{n}$ \\
Cbenopodium spp & 39 & 3 & Octubre $-3^{\circ}$ \\
Gramineas & 27 & 2 & Octubre $-3^{\circ}$ \\
Eucalipto & 10 & - & - \\
\hline
\end{tabular}

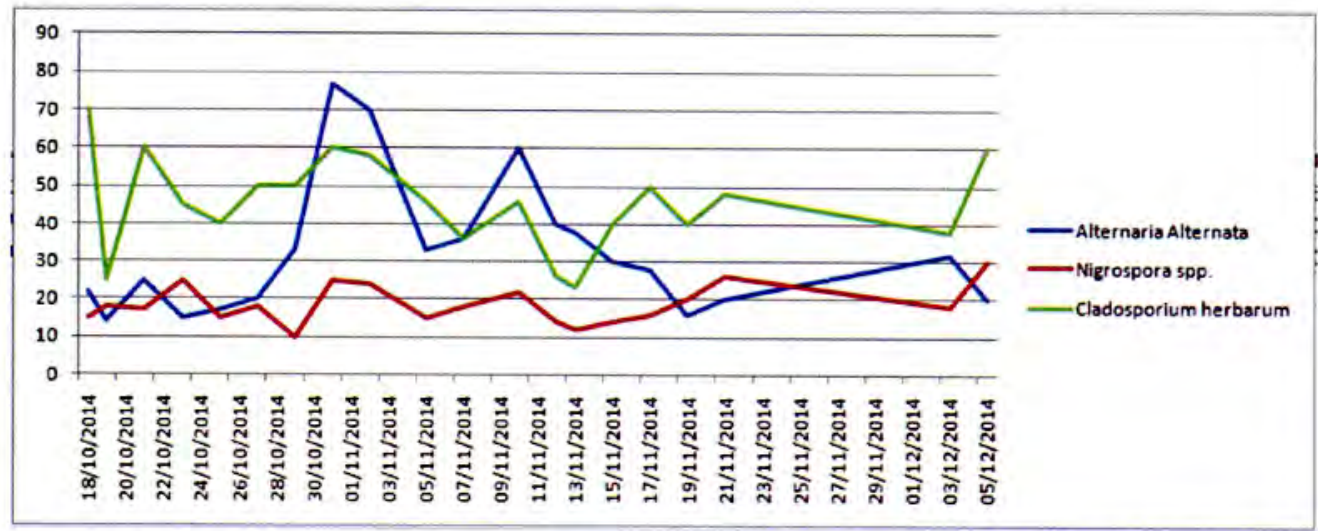

Figura 1. Conteo de Esporas en Tacna (Perú) durante la estación primaveral 2014. 
Calderón, $\mathbf{O}$. et al. Sensibilización y exposición a pólenes y esporas de hongos alergénicos aerotransportados en ciudades del sur de Perú.

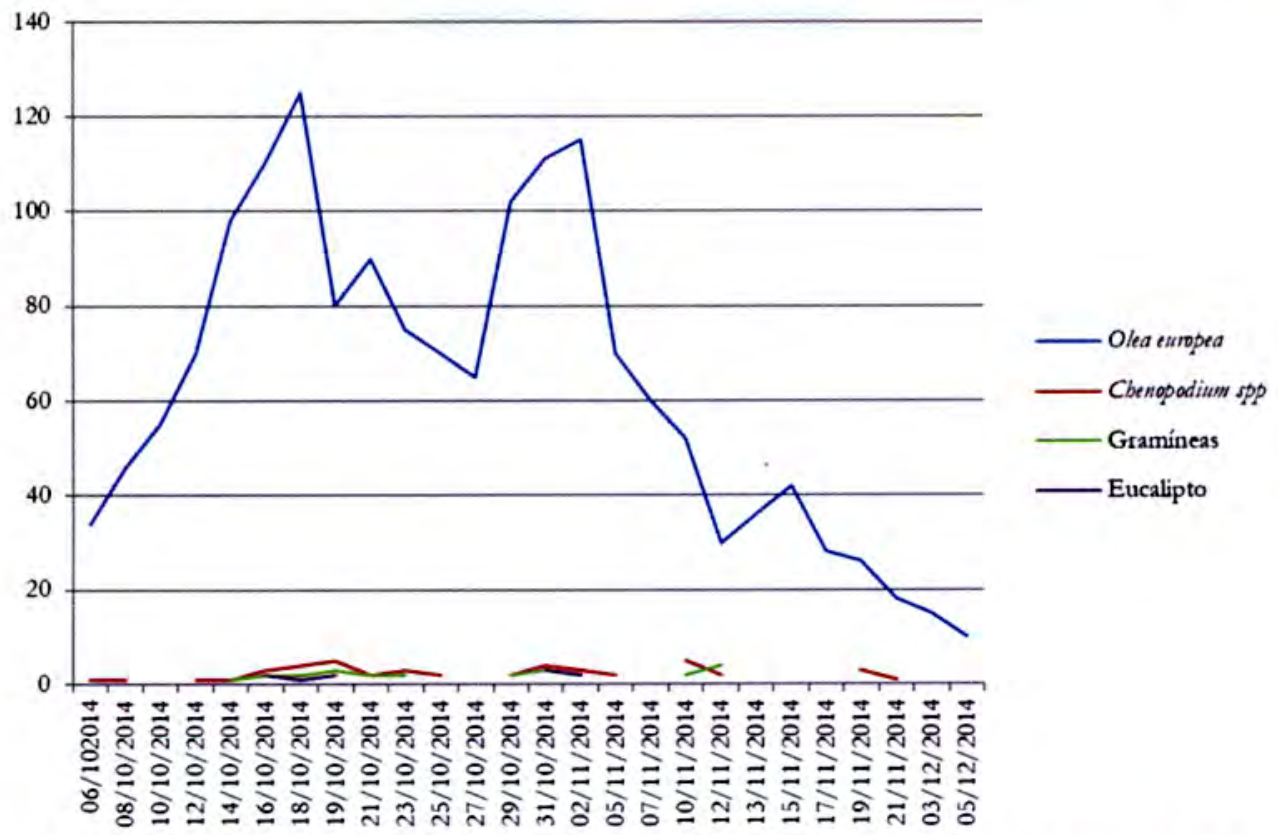

Figura 2. Conteo de granos de polen en Tacna (Perú) durante la estación primaveral 2014.

\section{DISCUSIÓN}

Fn los últimos años Perú ha incrementado su producción agrícola de olivos, siendo actualmente el primer productor en Latinoamérica, con un gran número de hectáreas cultivadas $(28000)$. Esta gran producción está localizada en la región sur de Perú (Ica, Pisco, Arequipa, especialmente en la ciudad de Tacna con 16000 hectáreas), esta última posee un área agrícola apropiada con condiciones climáticas favorables para la producción de olivos (clima templado con temperaturas en verano de $25-30^{\circ} \mathrm{C}$ y en invicrno $6-13^{\circ} \mathrm{C}$, lluvias anuales por debajo de $\operatorname{los} 100 \mathrm{~mm} y$ una humedad relativa de $75 \%$ ).

El polen de Otea europaea es mundialmente conocido por su alergenicidad como causante de rinoconjuntivitis $y / o$ asma en sujetos sensibilizados, como lo descrito en población de países mediterráneos en época de primavera.

Este estudio piloto nos ha llevado a observar un amplio perfil de sensibilización al polen de Olea europaea, en muestras alcatorias en las ciudades de Tacna (40\%) y Arcquipa $(36 \%)$, quienes están expuestos a altas concentraciones al polen de Olea europaea en los meses de primavera especialmente octubre, como lo descrito en otros países con una gran producción de olivo con los consecuentes altos niveles de granos de polen de Olea europaea por metro cúbico. ${ }^{1,23,45,7.8}$

Observamos personas con pruebas cutáneas positivas al polen de Olea europaea, con o sin sintomatología asociada en primavera. Por lo que, esta población en estudio podría estar genéticamente predispuesta, primero se sensibiliza al polen de Olea europaea y posteriormente desarrolla la sintomatología alérgica, como lo descrito en publicaciones cspanolas.

Por otro lado, encontramos también picos de esporas de Alternaria alternata, otro aeroalérgeno de mucha importancia como desencadenante de rinoconjuntivitis $y / o$ asma. Hallando importantes concentraciones en la atmósfe- ra como lo descrito por Bartra et $a l^{12}$ en Cataluña (España) y su relación con patología alérgica.

Éste es el primer estudio realizado en Perú sobre sensibilización y concentraciones de granos de polen y esporas de hongos medidos por método volumétrico. Sugerimos ampliar estudios que provean mayor información y sirvan como guía para una mejor prevención, diagnóstico y tratamiento para la población de la zona sur de Perú con enfermedades alérgicas, en este caso específico sobre el polen de Olea europaea y esporas de Alternaria alternata, 2 alérgenos aerotransportados de suma importancia y reconocidos mundialmentc. Además sc debcrían implementar estaciones de aerobiología como se hace en algunos países de Sudamérica y Europa, con la finalidad de investigar la variabilidad estacional y anual de pólenes y esporas de hongos alcrgénicos en esta zona, y posteriormente con estos resultados desarrollar estudios de sensibilización con los aeroalérgenos identificados en las ciudades.

\section{CONCLUSIONES}

El incremento del número de hectáreas de árboles de olivo cultivadas en la zona sur de Pcrú y por tanto las clevadas concentraciones de polen de Olea europaea al cual la población está expuesta, son actualmente un problema de salud pública a tener en cuenta por el desarrollo de enfermedades respiratorias alérgicas y sus consecuencias asociadas. La producción olivera del sur peruano continúa en aumento por su reconocida calidad a nivel internacional, esto conllevará un incremento de las patologías alérgicas asociadas, por lo que sc sugicre implementar programas de prevención, diagnóstico y tratamiento específico para mejorał la calidad de vida en los pacientes afectados. 


\section{REFERENCIAS BIBLIOGRÁFICAS}

1. Bousquet J, Heinzerling I, Bachert C, Papadopoulos NG, Bousquet PJ, Burney PG, et al. Practical guide to skin prick test in allergy to aeroallergens. Allergy. 2012;67 (1):18-24.

2. I.iccardi G, D' Amato M, D'Amato G. Oleaceae pollinosis: a revicw. Int Arch Allergy Immunol. 1996 Nov; 111(3):210-7.

3. Subiza J, Jerez M, Jiménez JA, Narganes MJ, Cabrera M, Varela S, Subiza E. Airborne pollen and polinosis in Madrid. I Allergy Clin Immunol 1995; 96:15-23.

4. D'Amato G, I obefalo G. Allergenic pollens in the southern Meditarranean area. J Allergy Clin Immunol, 1989; 83: 116-22.

5. Negrini, $\Lambda \mathrm{C}, \wedge$ robba D. Allergenic pollens and pollinosis in Italy: recent advances. A/lergy, 1992; 47:371-9.

6. Domínguez E, Infante F, Galán C, Guerra F, Villamandos F. Variations in the concentrations of airbone pollen asociated pollinosis in Córdoba (Spain): A study of the 10-year period 1982-91. J Invest Allergol Clin Immunol, 1993: 3 (3) 121-9.

7. Subiza J, Feo Brito F, Pola J, Moral A, Fernández J, Jerez M, Ferreiro M. Pólenes alergénicos y polinosis en 12 ciudades cspañolas. Rev Esp Alergol Inmunol Clin, 1998; 13:4563.

8. Florido JF, Delgado PG, de San Pedro BS, Quiralte J, de Saavedra JM, Peralta V, Valenzuela LR. High levels of Olea europaea pollen and relation with clinical findings. Int Arch Allergy Immunol. 1999 Jun; 119(2):133-7.

9. Barber D, Moreno C, Ledesma $\Lambda$, Serrano P, Galán $\Lambda$, Villalba M, Guerra F, Lombardero M, Rodríguez R. Degree of olive pollen exposure and sensitization patterns. Clinical implications. J Investig Allergol Clin Immunol. 2007;17 Suppl 1:11-6.

10. Cardaba B, Cortegano I, Florido F, Arrieta I, Aceituno E, del Pozo V, Gallardo S, Rojo M, Palomino P, Lahoz C. Genetic restrictions in olive pollen allergy. I Allergy Clin Immunol. 2000 Feb; 105:292-8.

11. B. Cárdaba, I. Cortegano, F. Florido1, E. Civantos, V. del Pozo, S. Gallardo, M. Rojo,P. Palomino, C. Lahoz. Update in the understanding of genetic predisposition to olive pollen sensitization. Allergy 2002: 57: Suppl. 71: 41-46.

12. Aguerri M, Calzada D, Montaner D, Mata M, Florido F, Quiralte J, Dopazo J, Lahoz C, Cardaba B. Differential gene-expression analysis defines a molecular pattern related to olive pollen allergy. J Biol Regul Homeost Agents. 2013 Apr-Jun; 27(2):337-50.

13. Bartra Tomás J. Aerobiological atmospheric spores of Alternaria alternata and sensitization prevalence on asthmatic and rbinitic population in Catalunya. (Doctoral thesis). Catalunya - Spain. Universidad Autónoma de Barcelona. 2004.

\section{Correspondencia:}

Oscar Manuel Calderón Llosa: oscarcalderonll@gmail.com

Silvia Antonieta Uriarte Obando: silvia_uriarte@hotmail.com

Gregorio Pedro Tejada Monroy.montepinal@hotmait.com
Fecha de Recepción: 16/03/2015

Fecha de Aceptación: 09/06/2015 